\title{
Nerve transfers for restoration of finger flexion in patients with tetraplegia
}

\author{
Jayme A. Bertelli, MD, PhD, ${ }^{1,2}$ and Marcos F. Ghizoni, MD, MSc ${ }^{2}$ \\ ${ }^{1}$ Center of Biological and Health Sciences, Department of Neurosurgery, University of the South of Santa Catarina (Unisul), \\ Tubarão; and 'Department of Orthopedic Surgery, Governador Celso Ramos Hospital, Florianópolis, Santa Catarina, Brazil
}

OBJECTIVE The purpose of this paper was to report the authors' results with finger flexion restoration by nerve transfer in patients with tetraplegia.

METHODS Surgery was performed for restoration of finger flexion in 17 upper limbs of 9 patients (8 male and 1 female) at a mean of 7.6 months (SD 4 months) after cervical spinal cord injury. The patients' mean age at the time of surgery was 28 years (SD 15 years). The motor level according to the ASIA (American Spinal Injury Association) classification was C-5 in 4 upper limbs, C-6 in 10, and C-7 in 3.

In 3 upper limbs, the nerve to the brachialis was transferred to the anterior interosseous nerve (AIN), which was separated from the median nerve from the antecubital fossa to the midarm. In 5 upper limbs, the nerve to the brachialis was transferred to median nerve motor fascicles innervating finger flexion muscles in the midarm. In 4 upper limbs, the nerve to the brachioradialis was transferred to the AIN. In the remaining 5 upper limbs, the nerve to the extensor carpi radialis brevis (ECRB) was transferred to the AIN. Patients were followed for an average of 16 months (SD 6 months). At the final evaluation the range of finger flexion and strength were estimated by manual muscle testing according to the British Medical Research Council scale.

RESULTS Restoration of finger flexion was observed in 4 of 8 upper limbs in which the nerve to the brachialis was used as a donor. The range of motion was incomplete in all 5 of these limbs, and the strength was M3 in 3 limbs and M4 in $1 \mathrm{limb}$. Proximal retrograde dissection of the AIN was associated with better outcomes than transfer of the nerve to the brachialis to median nerve motor fascicles in the arm. After the nerve to the brachioradialis was transferred to the AIN, incomplete finger flexion with M4 strength was restored in $1 \mathrm{limb}$; the remaining 3 limbs did not show any recovery. Full finger flexion with M4 strength was demonstrated in all 5 upper limbs in which the nerve to the ECRB was transferred to the AIN. No functional downgrading of elbow flexion or wrist extension strength was observed.

CONCLUSIONS In patients with tetraplegia, finger flexion can be restored by nerve transfer. Nerve transfer using the nerve to the ECRB as the donor nerve produced better recovery of finger flexion in comparison with nerve transfer using the nerve to the brachialis or brachioradialis.

http://thejns.org/doi/abs/10.3171/2016.5.SPINE151544

KEY WORDS spinal cord injury; nerve transfer; tetraplegia

$\mathrm{A}$ PPROXIMATELY 11,000 spinal cord injuries occur each year in the United States. ${ }^{1}$ In underdeveloped countries the number of new spinal cord injuries per million persons in the population is likely to be greater than in developed countries. In Brazil, it is estimated that $8.6 \%$ of all beds in public hospitals are occupied by patients with spinal cord injuries, and approximately 11,000 new cases occur each year. ${ }^{22,26}$ These injuries are occurring with increasing frequency even in developed countries. ${ }^{18,25}$ Car accidents, gunshot injuries, and falls in gen- eral are the predominant causes of spinal cord trauma. ${ }^{26}$ Most individuals affected are young men (21-30 years of age), and in at least half of these cases the injury is to the cervical spinal cord. ${ }^{10,26}$ Cervical spinal cord injuries can result in tetraplegia with severely compromised function of the upper limbs in addition to paralysis of the trunk and lower extremities. Affected patients often survive for a long time, with the estimated survival ranging from 23 to 40 years according to different studies ${ }^{25}$ Cure of paralysis is still not possible because of the inherent problems of

ABBREVIATIONS AIN = anterior interosseous nerve; $\mathrm{ASIA}=$ American Spinal Injury Association; $\mathrm{ECRB}=$ extensor carpi radialis brevis; ECRL = extensor carpi radialis longus; ICSH = International Classification for Surgery of the Hand; ISNCSCI = International Standards for Neurological Classification of Spinal Cord Injury.

SUBMITTED December 24, 2015. ACCEPTED May 12, 2016.

INCLUDE WHEN CITING Published online August 5, 2016; DOI: 10.3171/2016.5.SPINE151544. 
nerve regeneration in the central nerve system..${ }^{10}$ Because cure is not possible, rehabilitation has focused on training of compensation techniques. ${ }^{10}$ In a recent survey, $50 \%$ of 347 patients with tetraplegia indicated that recovery of $\mathrm{arm} /$ hand function would be crucial to improve their quality of life. Another $11 \%$ of the patients ranked increased upper body/trunk strength and balance as most important. Regaining "walking movement" was the highest priority for only $8 \%$ of the patients. ${ }^{1}$

Reconstructive upper-extremity tendon transfer surgeries emerged in the 1950 s to optimize function by improving elbow extension and grip and pinch strength. ${ }^{23}$ Following surgery, range of motion and grip and pinch strength may improve considerably, and individuals may complete daily living and leisure activities more quickly and efficiently. ${ }^{14,15}$ Despite documented improvements, tendon transfer surgery is offered to and finally performed in a very restricted number of patients. ${ }^{24}$ It is possible that the need for several surgeries and immobilization periods limit physiotherapists' recommendations and patients' acceptance for tendon transfer surgery.

More recently the use of distal nerve transfers has emerged as an adjunct therapy to improve upper limb function in patients with tetraplegia. ${ }^{6,12}$ In this strategy, by crossing peripheral nerves, axons from motoneurons that originate above the lesion level and have functional cortical drive are rerouted to reinnervate target muscles that are normally innervated by motoneurons located below the spinal cord lesion. In patients with midcervical spinal cord injuries, distal nerve transfer resulted in predictable restoration of elbow and finger extension with a single surgery, no postoperative casting, and only 1 day of hospitalization. ${ }^{6}$

We now report our results for restoration of finger flexion using nerve transfers in patients with tetraplegia.

\section{Methods}

The ethics committee of the University of the South of Santa Catarina (Unisul) approved the protocol of the clinical trial in advance of any data collection. Patients provided verbal informed consent before participation, in accordance with the Declaration of Helsinki.

Between July 2011 and December 2013 a consecutive series of 9 patients ( 8 male and 1 female; mean age 28 years, SD 15 years) underwent bilateral single surgery for restoration of upper limb function. In 17 upper limbs, elbow, thumb, and finger extension together with finger flexion were restored via nerve transfers. In one of the 18 upper limbs only elbow and thumb and finger extension were restored via nerve transfers. In this upper limb, finger flexion was not restored because of poor donor nerves. We opted for later reconstruction of finger flexion by a tendon transfer. All patients had complete spinal cord lesions (American Spinal Injury Association [ASIA] Impairment Scale Grade A) as a result of road traffic accidents, and all had undergone surgery for spine stabilization and/or spinal cord decompression during the 1st week after the accident. Surgery for global upper limb restoration was performed at a mean of 7.6 months (SD 4 months) after the accident. None of the patients had traumatic brain injury or cogni- tive impairment following the accident. In 4 upper limbs, the motor level of injury was C-5 according to International Standards for Neurological Classification of Spinal Cord Injury (ISNCSCI), ${ }^{10}$ and all 4 limbs met the criteria for Group 1 of the International Classification for Surgery of the Hand (ICSH).$^{10}$ In 10 of the 17 upper limbs in which nerve transfer was performed for restoration of finger flexion, the motor level of injury was C-6. In 3 of these 10 upper limbs, both the extensor carpi radialis brevis (ECRB) and the extensor carpi radialis longus (ECRL) were functional and these upper limbs were scored as ICSH Group 4. In 3 other upper limbs, the motor level of injury was C7; their ICSH classifications were Group 3 in 1 case and Group 7 in 2 cases. We used clinical examination based on tendon palpation to determine the status of the radial wrist extensors. To test the ECRL, the forearm was pronated, while the wrist was extended to $20^{\circ}$ and deviated toward the ulna with the fingers flexed. The examiner resisted radial deviation while palpating the tendon of the ECRL at the base of the second metacarpal. The ECRB tendon was palpated at the base of third metacarpal during resisted wrist extension with the wrist pronated and in $20^{\circ}$ of extension. If, during the proposed testing maneuver, the examiner's fingertip moved 3-4 mm, as detected by palpation and visual assessment, despite resisting motion, the tested muscle was deemed preserved. Details of the clinical examination of wrist extensors can be obtained elsewhere. ${ }^{3}$

Surgery was performed under general anesthesia without muscle relaxants. For finger flexion restoration, the recipient nerve was the anterior interosseous nerve (AIN) in 9 limbs or median nerve motor fascicles related to the AIN at the midarm in 8 limbs. The donor nerve was the nerve to the brachialis muscle in 8 limbs, the nerve to the brachioradialis muscle in 4 limbs, and the nerve to the ECRB in 5 limbs. When the ECRB was functional it was our first choice as a donor for transfer. When the ECRB was paralyzed, we used either the nerve to the brachialis or the nerve to the brachioradialis, with no further specific criteria for donor nerve selection. No randomization was employed in this study.

In 3 limbs in which we used the nerve to the brachialis as the donor nerve for transfer to the AIN, we made a long incision from the upper third of the arm to the antecubital fossa (Fig. 1). In the arm, we located the musculocutaneous nerve, the biceps motor branch, and the nerve to the brachialis, which was dissected as distally as possible. In the elbow, we located the AIN and dissected it in a retrograde fashion to a point where coaptation with the nerve to the brachialis was possible without tension. This necessitated extended dissections of approximately 15-18 $\mathrm{cm}$ within the median nerve. In no instance was there a clear anatomic plane of separation of the AIN from other fascicles of the median nerve. In the remaining 5 limbs, to minimize surgery by avoiding extended dissection of the AIN, we selected recipient motor fascicles within the median nerve by electric stimulation. Because many AIN motoneurons are located below the site of the spinal cord lesions, despite paralysis following interruption of supraspinal control, median nerve fascicles forming the AIN do not undergo Wallerian degeneration and electric stimulation can reveal those fascicles aiming at thumb and finger flexion. Through a $10-\mathrm{cm}-$ long incision in the upper arm 

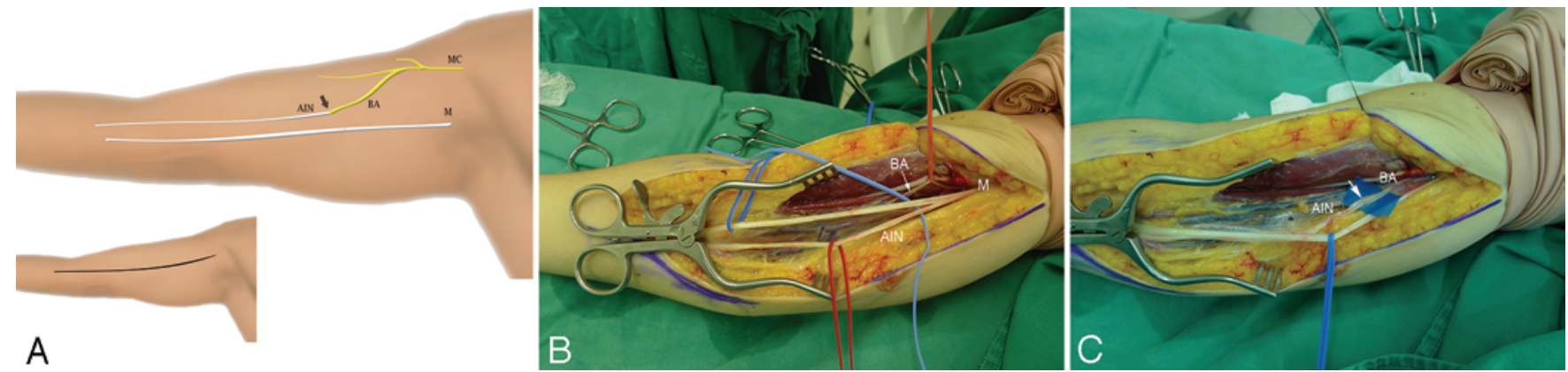

FIG. 1. A: Schematic representation of the skin incision and transfer of the nerve to brachialis (BA) to the AIN after retrograde dissection of the latter. $\mathrm{M}=$ median nerve; $\mathrm{MC}=$ musculocutaneous nerve. The arrow indicates the site of nerve coaptation. B: Intraoperative view of the right upper limb (Case 9) following dissection of the AIN from the median nerve (M). C: Intraoperative view of the right upper limb (Case 9) after coaptation (arrow) with the nerve to the brachialis (BA). Figure is available in color online only.

we dissected the nerve to the brachialis and the median nerve. On the medial side of the median nerve we performed a longitudinal 15 -mm-long epineurotomy and separated the nerve fascicles. One or 2 posteromedially located fascicles that induced flexion of thumb and fingers following electrical stimulation were sectioned and coapted with the nerve to the brachialis (Fig. 2).

When we used the nerve to the brachioradialis muscle as the donor for nerve transfer to the AIN, we used a Y incision centered on the antecubital fossa (Fig. 3). The medial limb of the Y incision allowed us to dissect the AIN proximally within the median nerve, whereas the lateral limb was used to locate the nerve to the brachioradialis. Proximal dissection of the AIN was always necessary to permit direct coaptation with the nerve to the brachioradialis. For coapting the ECRB with the AIN (Fig. 4), we used an oblique incision placed on the antecubital fossa as previously described. ${ }^{9}$

In all cases, we used intraoperative electrical stimulation to confirm the identity and health of donor and recipient nerves. For electrical stimulation, we used an insulated 21-gauge needle (Contiplex D; B. Braun Melsungen AG) connected to a nerve stimulator (Stimuplex HNS 11; B.
Braun Melsungen AG). We performed all nerve coaptation under the microscope using 9-0 monofilament sutures.

After surgery, an arm sling was used for 7-10 days. Patients received no special physiotherapy training. They received 4 cycles of 5 doses of nandrolone $(50 \mathrm{mg}$ for the male patients and $25 \mathrm{mg}$ for the 1 female patient) administered intramuscularly once every 15 days, with an interval of 30 days between cycles, as previously reported ${ }^{6}$

At the last follow-up visit, which occurred an average of 16 months after surgery, we evaluated the range of finger flexion and strength as per British Medical Research Council guidelines. ${ }^{16}$ We defined complete flexion as all fingertips touching the palm of the hand with the wrist at neutral to avoid any tenodesis effect promoted by wrist extension. We deemed as "failure" any case in which strength of at least M3 was not achieved.

A summary of the demographic and clinical characteristics of the 9 patients in this series is shown in Table 1.

\section{Results}

We observed recovery of M3 or better finger flexion strength in 10 of the 17 surgically treated upper limbs.
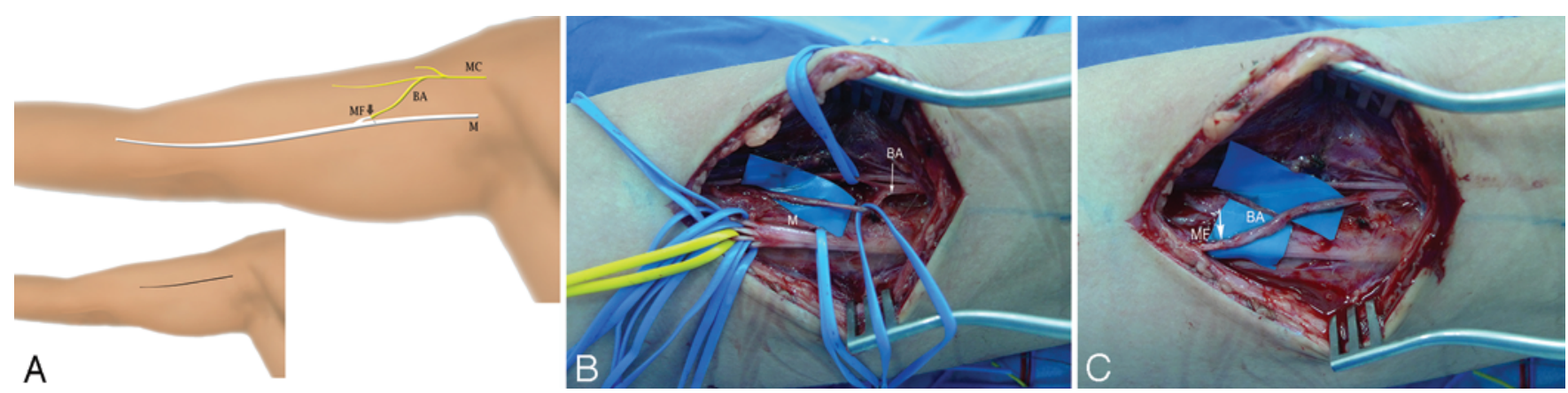

FIG. 2. A: Schematic representation of the skin incision and transfer of the nerve to the brachialis (BA) to median nerve motor fascicles (MF) aimed at finger flexion demonstrated by intraoperative electric stimulation. $\mathrm{M}=$ median nerve; $\mathrm{MC}=$ musculocutaneous nerve. The arrow indicates the site of nerve coaptation. B: Intraoperative view of the right midarm region (Case 4) after dissection of median nerve (M) motor fascicles and the nerve to the brachialis (BA). The nerve to the brachialis is dissected as distally as possible within the brachialis muscle. C: Intraoperative photograph showing coaptation of the nerve to the brachialis (BA) to median nerve motor fascicles (MF) aimed at innervation of the flexor digitorum profundus and flexor pollicis longus identified after electric stimulation (Case 4). The arrow indicates the site of nerve coaptation. Figure is available in color online only. 

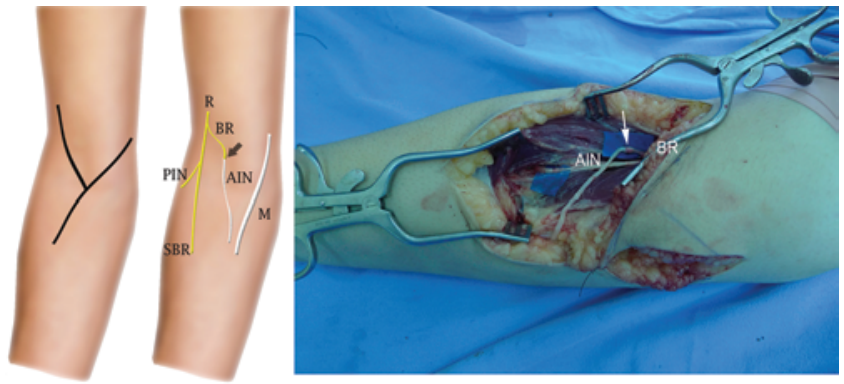

FIG. 3. Left: Schematic representation of the skin incision and transfer of the nerve to the brachioradialis (BR) to the AIN. $M=$ median nerve; $\mathrm{PIN}=$ posterior interosseous nerve; $\mathrm{R}=$ radial nerve; $\mathrm{SBR}=$ superficial branch of the radial nerve. The arrow indicates the site of nerve coaptation. Right: Intraoperative view of the right antecubital fossa showing the connection of the AIN with the nerve to the brachioradialis (NBR) (Case 5). The arrow indicates the site of nerve coaptation. Figure is available in color online only.

Failures were associated with either transfer of the nerve to the brachialis to motor fascicles of the AIN at the proximal arm or with transfer of the nerve to the brachioradialis to the AIN. There were no failures in cases in which the nerve to the brachialis was connected to the proximally dissected AIN or when the nerve to the ECRB was transferred to the AIN.

In 4 of 8 upper limbs, use of the nerve to the brachialis as the donor nerve resulted in restoration of active finger flexion. No patient demonstrated complete range of finger flexion. Of the 3 limbs in which the proximally dissected AIN was the recipient nerve, 2 had M3 strength and 1 had M4 strength. There was no failure. Of the 5 upper limbs in which the motor fascicles of the AIN in the median nerve in the proximal arm served as the recipient, 1 had M3 strength whereas failure of recovery was observed in the remaining 4 upper limbs. After transferring the nerve to the brachioradialis to the AIN (4 limbs) we observed recovery of partial range of finger flexion range of motion scoring M4 in 1 upper limb. There was no recovery in the remaining 3 upper limbs. Transfer of the ECRB motor branch to the AIN resulted in complete finger flexion with M4 strength in all 5 upper limbs. Videos 1-4 show preand postsurgery footage of representative cases.

VIDEO 1. Case 1. Video clip showing postoperative footage. $M=$ median nerve; $\mathrm{MF}=$ motor fascicles of the median nerve aimed at the flexor digitorum profundus and flexor pollicis longus (i.e., AIN); PIN = posterior interosseous nerve; $R=$ radial nerve; $S B R=$ superficial branch of the radial nerve. Copyright Jayme A. Bertelli. Published with permission. Click here to view.

VIDEO 2. Case 2. Video clip showing pre- and postoperative footage. Copyright Jayme A. Bertelli. Published with permission. Click here to view.

VIDEO 3. Case 8. Video clip showing pre- and postoperative footage. $\mathrm{M}=$ median nerve; $\mathrm{PIN}=$ posterior interosseous nerve; $\mathrm{R}=$ radial nerve; SBR = superficial branch of the radial nerve. Copyright Jayme A. Bertelli. Published with permission. Click here to view.

VIDEO 4. Case 9. Video clip showing pre- and postoperative footage. $\mathrm{M}=$ median nerve; $\mathrm{PIN}=$ posterior interosseous nerve. Copyright Jayme A. Bertelli. Published with permission. Click here to view.

After proximal dissection of the AIN for connection with the nerve to the brachialis, all patients complained about numbness on the radial side of the hand; this numbness disappeared within 3 months after surgery. Elbow flexion strength was preserved in all patients in whom either the nerve to the brachialis or the nerve to the brachioradialis was harvested for transfer. Wrist extension power was not downgraded in any patient in whom the motor branch of the ECRB was used; all scored M4 in wrist extension strength.

The results with respect to restoration of finger flexion are summarized in Table 1.

\section{Discussion}

\section{Restoration of Finger Flexion}

Transfer of the Nerve to the Brachialis to the AIN

After epineurotomy and single fascicular electric stimulation, similarly to Mackinnon et al., ${ }^{20}$ we found motor fascicles of the AIN in the posteromedial quadrant of the
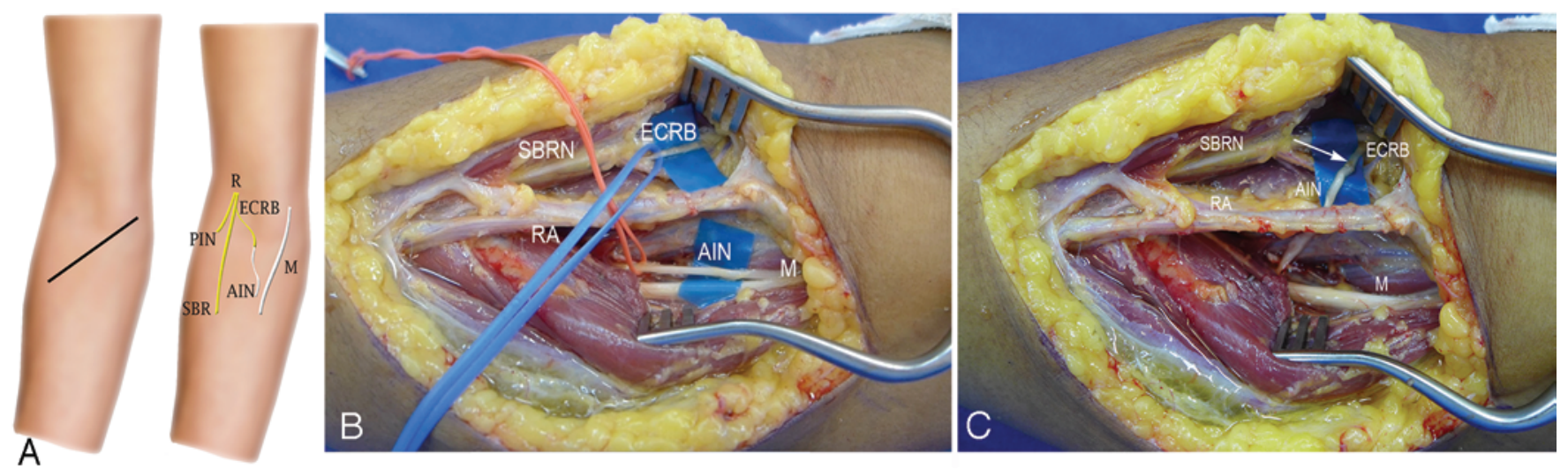

FIG. 4. A: Schematic representation of the skin incision and transfer of the nerve to the ECRB to the AIN. M = median nerve; PIN $=$ posterior interosseous nerve; $R=$ radial nerve; $S B R=$ superficial branch of the radial nerve. The arrow indicates the site of nerve coaptation. B: Intraoperative view of the right antecubital fossa prior to nerve transfer. Note the superficial branch of the radial nerve (SBRN), the nerve to the ECRB, the radial artery (RA), the AIN and the median nerve (M) (Case 8). C: The nerve to the ECRB is transferred to the AIN (Case 8). The arrow indicates the site of nerve repair. Figure is available in color online only. 


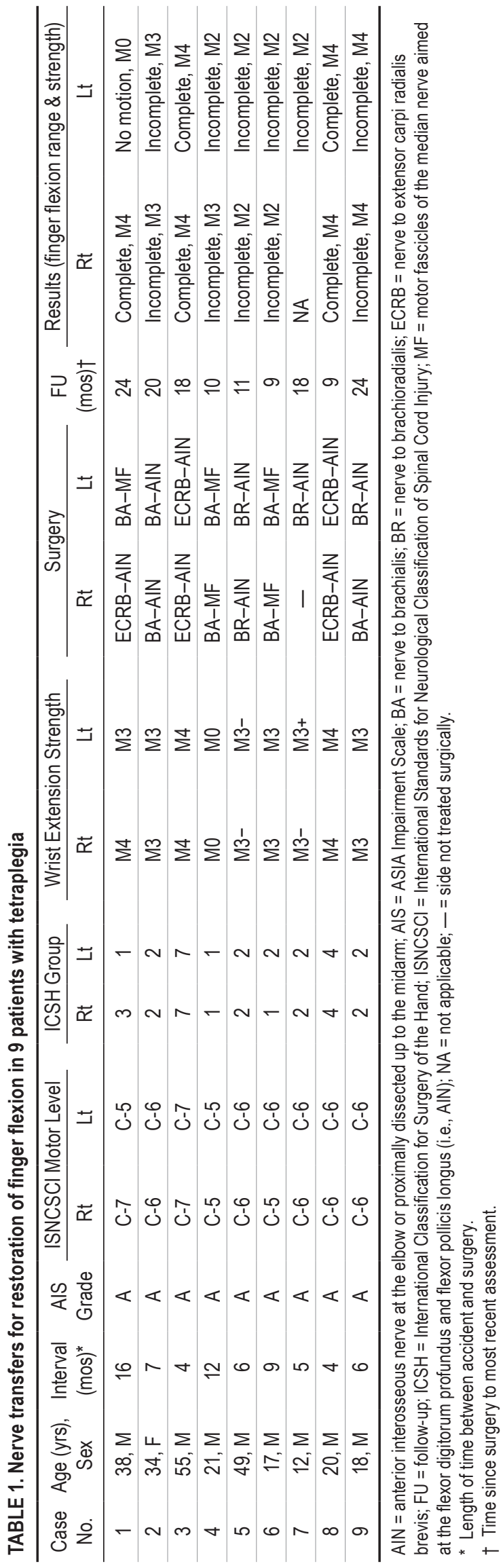

median nerve. This differs from the findings of Sunderland, ${ }^{29}$ based on cadaveric dissection, and Pham et al., ${ }^{27}$ based on MRI findings in patients with AIN palsy. Sunderland ${ }^{29}$ and Pham et al. ${ }^{27}$ indicated that motor axons of the AIN are located in the posterolateral quadrant of the median nerve. Differences between these findings are probably related to the method used to determine the location of the AIN fascicles within the median nerve.

Transfer of the branch to the brachialis to the AIN or to median nerve motor fascicles resulted in M3 strength recovery in 3 limbs and M4 in one limb. We identified 4 failures (i.e., M0-M2 strength). We speculate that failures and inconsistency of M4 strength recovery might be related to 5 possible causes, including 1) short duration of follow-up, 2) the distance of the nerve coaptation being very far (more than $15 \mathrm{~cm}$ ) from the motor endplates of the finger flexors, and 3) the plexiform disposition of the median nerve fascicles. Interconnections between fascicles of the median nerve might induce axonal growth dispersion. We have previously suggested that the internal architecture of nerve trunks might be deleterious for axonal regrowth. ${ }^{7}$ Proximal dissection of the AIN was associated with better outcomes, presumably because section of nerve fiber interconnections during dissection section resulted in a more direct axonal regrowth, thus decreasing axonal dispersion. A fourth possible reason for the observed failures and inconsistency of M4 strength recovery could be the scattered disposition of motor fascicles within the median nerve. At the root of the limb, connection of the nerve to the brachialis to fascicles aimed at finger flexion does not address all fibers of the AIN. This might account for partial reinnervation of the finger flexors. This scattered disposition of motor fascicles allows fascicle harvesting for transfer without causing a functional downgrade of finger flexion strength. ${ }^{30}$ In this connection, dispersion of motor fibers within the median nerve might be responsible for spontaneous recovery and improved results after nerve transfer using the nerve to the brachialis as the donor nerve and the AIN as the recipient. ${ }^{32}$ The fifth possible cause involves limited specificity of electric stimulation for recipient motor fascicles selection within the median nerve. In upper root brachial plexus injuries, for elbow flexion reconstruction, after intraoperative electric stimulation, motor fascicles of the ulnar nerve are transferred to the biceps motor branch. ${ }^{5}$ After reinnervation, we observed that squeezing the biceps muscle produced numbness in the ulnar fingers, indicating that our transferred fascicles were not pure motor fascicles. ${ }^{2}$ In fact, at the root of the limb, all nerve fascicles are mixed. ${ }^{4}$ Possibly many motor axons from the nerve to the brachialis got misrouted into sensory pathways during regeneration. Some surgeons have suggested that motor axons preferentially reinnervate sensory pathways. ${ }^{4,21}$

Fox et al. ${ }^{12}$ have also observed the absence of M4 finger flexion strength recovery following transfer of the nerve to the brachialis to median nerve motor fascicles in the arm. In contrast, Krasuski and Kiwerski ${ }^{19}$ observed M4 finger flexion strength recovery in 16 of 42 tetraplegic patients in whom the branch to the brachialis and the lateral antebrachial cutaneous nerve were transferred to the median nerve. After a minimum follow-up of 24 months, they observed that good results were clearly associated with age 
of less than 25 years and surgery within 3 months after accident. The fact that we have not addressed the entire median nerve, the longer interval between accident and surgery, and greater patient age may explain our lack of M4 strength recovery in 7 of 8 limbs. In the single case in which we observed M4 finger flexion recovery, the patient was 18 years old at the time of surgery and the surgery was performed 6 months after his accident. In midcervical spinal cord lesions, distal cutaneous sensation is better preserved than distal motor function, which implicates sensory preservation on the territory of the median nerve. ${ }^{33}$ Therefore, in our opinion the major drawback of the surgery described by Krasuski and Kiwerski ${ }^{19}$ is disturbing hand sensation by total division of the median nerve.

\section{Transfer of the Nerve to the Brachioradialis to the AIN}

Despite nerve coaptation close to the motor endplates, use of the nerve to the brachioradialis for transfer to the AIN resulted in poor outcomes, with only a single instance of M4 strength recovery among 4 limbs, and even that single instance resulted in incomplete range of finger flexion. The poor outcomes may be related to 2 factors. 1 ) The number of motor axons in the nerve to the brachioradialis may be insufficient for AIN neurotization. The mean number of myelinated fibers in the nerve to the brachioradialis was reported to be $550 \pm 64$ in an anatomic study, whereas the corresponding number in the AIN was $2266 \pm 274 .{ }^{13}$ Hence, myelinated fibers within the nerve to the brachioradialis correspond to only $24 \%$ of those in the AIN. Ideally, the number of myelinated fibers in the donor nerve should be at least $70 \%$ of the number in the recipient nerve..$^{28}$ 2) Partial motoneuron loss may occur after a spinal cord injury, and this would decrease muscle strength as well as the number of nerve fibers available for transfer. In the concomitant paralysis of the radial wrist extensors, the brachialis may be partially denervated ${ }^{17}$ It is important to note that it is difficult to determine brachioradialis muscle strength preoperatively. The brachioradialis is an elbow flexor, and it cannot be tested separately from the biceps and brachialis muscles. In general, it is tested by palpation of its belly during elbow flexion, but this is only an indirect measure of strength. ${ }^{17}$

\section{Transfer of the Nerve to the ECRB to the AIN}

We observed complete finger flexion with M4 strength in all 5 limbs in which the nerve to the ECRB was transferred to the AIN. These good outcomes resulted from nerve coaptation close to the motor endplates, with axons navigating from one to another pure motor nerve. When the fingers are flexing, the ECRB co-contracts to stabilize the wrist, avoiding concomitant wrist flexion provoked by the contraction of finger flexors. The more strongly the fingers flex, the more strongly the ECRB co-contracts. ${ }^{3}$ This synergistic action of the ECRB with finger flexors possibly favored functional recovery. This concept of synergistic co-contraction is totally different from the established axiom that wrist extension promotes finger flexion. In the latter concept, wrist extension promotes secondary finger flexion mechanically, by increasing the distance between the origin of the long flexors and their insertion. ${ }^{33} \mathrm{We}$ observed recovery of finger flexion in all fingers, including those innervated by the ulnar nerve in 4 of 5 upper limbs.
Possibly this occurred because of intermuscular nerve connections between the ulnar nerve and AIN branches and because of flexor digitorum profundus tendinous interconnections. ${ }^{8}$ After harvesting the branch of the radial nerve to the ECRB for transfer, wrist extension, not associated with radial deviation, was preserved in all our patients. This possibly results from preservation or reinnervation of the extensor carpi ulnaris following transfer of the nerve to supinator to the posterior interosseous nerve. Unfortunately, the ECRB might be paralyzed and not available for transfer in a great number of patients with tetraplegia. Our clinical method for testing the ECRB and ECRL by tendon palpation $^{3}$ was reliable because of the clinical recovery of finger flexion and preservation of wrist extension after harvesting of the nerve to the ECRB. After harvesting of the radial nerve branch to the ECRB, in case of failure of the nerve transfer, the ECRL would not be suitable as a donor to tendon transfer for finger flexion. In this situation, potential donors would be the brachioradialis and the brachialis muscles. ${ }^{4}$

\section{Peripheral Versus Central Nerve Palsy}

In cervical spinal cord injuries, central and peripheral parts of the motor system can be affected. ${ }^{13}$ Effects on the motoneuron pool and peripheral nerve system leads to a flaccid paralysis with muscle denervation and pronounced atrophy. Palsy resulting from a pyramidal tract lesion produces no muscle denervation, and consequently it is possible that delayed reinnervation is less important than in the peripheral type of palsy. ${ }^{11}$ In a previous study we identified a peripheral type of palsy in 22 of the 27 recipient nerves. ${ }^{6}$ In the present study, no peripheral type of palsy was demonstrated following AIN electrical stimulation. In both studies, the spinal cord injuries were located at the C- 6 level or above. The differences between these 2 studies might be related to muscle sampling. In the former study we sampled muscles with the motoneuron pool located above or at the spinal cord lesion site (i.e., the triceps), whereas in the present study we sampled muscles innervated by motoneurons that originate below the lesions (i.e., finger flexors). Similar findings concerning finger flexor muscles have been previously reported for electrophysiological studies in patients with tetraplegia due to cervical spinal cord injury. ${ }^{11}$

\section{Study Limitations}

Limitations of our study include the small number of patients and the fact that they underwent different surgical procedures. We did not base our indications for nerve transfer surgery on electrophysiological studies because electromyograms do not reflect either muscle strength or volitional control. ${ }^{31}$

We did not objectively study finger flexion strength recovery nor did we study the impact of finger flexion restoration in daily activities.

\section{Conclusions}

In patients with tetraplegia, finger flexion can be restored by nerve transfers. The ECRB is a better donor than the nerve to the brachialis or the nerve to the brachioradialis. 


\section{References}

1. Anderson KD: Targeting recovery: priorities of the spinal cord-injured population. J Neurotrauma 21:1371-1383, 2004

2. Bertelli JA: Letter regarding "The value of the tender muscle sign in detecting motor recovery after peripheral nerve reconstruction.” J Hand Surg Am 40:1919, 2015 (Letter)

3. Bertelli JA: Tendon palpation during agonist contraction and antagonist co-contraction to assess wrist flexor and extensor muscle function. Chir Main 34:79-85, 2015

4. Bertelli JA, Ghizoni MF: Brachialis muscle transfer to reconstruct finger flexion or wrist extension in brachial plexus palsy. J Hand Surg Am 31:190-196, 2006

5. Bertelli JA, Ghizoni MF: Nerve root grafting and distal nerve transfers for C5-C6 brachial plexus injuries. J Hand Surg Am 35:769-775, 2010

6. Bertelli JA, Ghizoni MF: Nerve transfers for elbow and finger extension reconstruction in midcervical spinal cord injuries. J Neurosurg 122:121-127, 2015

7. Bertelli JA, Ghizoni MF: Results of c5 root grafting to the musculocutaneous nerve using pedicled, vascularized ulnar nerve grafts. J Hand Surg Am 34:1821-1826, 2009

8. Bertelli JA, Ghizoni MF, Tacca CP: The median nerve consistently drives flexion of the distal phalanx of the ring and little fingers: Interest in finger flexion reconstruction by nerve transfers. Microsurgery 35:207-210, 2015

9. Bertelli JA, Taleb M, Mira JC, Ghizoni MF: Variation in nerve autograft length increases fibre misdirection and decreases pruning effectiveness: an experimental study in the rat median nerve. Neurol Res 27:657-665, 2005

10. Bryden A, Sinnott A, Mulcahey MJ: Innovative strategies for improving upper extremity function in tetraplegia and considerations in measuring functional outcomes. Top Spinal Cord Inj Rehabil 10:75-93, 2005

11. Curt A, Dietz V: Neurographic assessment of intramedullary motoneurone lesions in cervical spinal cord injury: consequences for hand function. Spinal Cord 34:326-332, 1996

12. Fox IK, Davidge KM, Novak CB, Hoben G, Kahn LC, Juknis $\mathrm{N}$, et al: Nerve transfers to restore upper extremity function in cervical spinal cord injury: update and preliminary outcomes. Plast Reconstr Surg 136:780-792, 2015

13. García-López A, Fernández E, Martínez F: Transfer of brachioradialis motor branch to the anterior interosseous nerve in C8-T1 brachial plexus palsy. An anatomic study. Microsurgery 33:297-300, 2013

14. Hamou C, Shah NR, DiPonio L, Curtin CM: Pinch and elbow extension restoration in people with tetraplegia: a systematic review of the literature. J Hand Surg Am 34:692699, 2009

15. Hentz VR, Brown M, Keoshian LA: Upper limb reconstruction in quadriplegia: functional assessment and proposed treatment modifications. J Hand Surg Am 8:119-131, 1983

16. James MA: Use of the Medical Research Council muscle strength grading system in the upper extremity. J Hand Surg Am 32:154-156, 2007

17. Johnson DL, Gellman H, Waters RL, Tognella M: Brachioradialis transfer for wrist extension in tetraplegic patients who have fifth-cervical-level neurological function. J Bone Joint Surg Am 78:1063-1067, 1996

18. Koskinen EA, Alen M, Väärälä EM, Rellman J, Kallinen M, Vainionpää A: Centralized spinal cord injury care in Finland: unveiling the hidden incidence of traumatic injuries. Spinal Cord 52:779-784, 2014

19. Krasuski M, Kiwerski J: An analysis of the results of transferring the musculocutaneous nerve onto the median nerve in tetraplegics. Arch Orthop Trauma Surg 111:32-33, 1991

20. Mackinnon SE, Yee A, Ray WZ: Nerve transfers for the restoration of hand function after spinal cord injury. J Neurosurg 117:176-185, 2012
21. Maki Y, Yoshizu T, Tajima T, Narisawa H: The selectivity of regenerating motor and sensory axons. J Reconstr Microsurg 12:547-551, 1996

22. Masini M: Estimativa da incidência e prevalência de lesão medular no Brasil. J Bras Neurocirug 12:97-100, 2001

23. Moberg E: Surgical treatment for absent single-hand grip and elbow extension in quadriplegia. Principles and preliminary experience. J Bone Joint Surg Am 57:196-206, 1975

24. Nguyen BH, Gagnon D, Danino AM, De Iure A, Robidoux I, Riley-Nobert M, et al: Development of a clinical algorithm to triage potential candidates for upper extremity tendon transfer surgery in individuals with tetraplegia: a retrospective study. Int J Phys Med Rehabil 2:176, 2014

25. O'Connor PJ: Prevalence of spinal cord injury in Australia. Spinal Cord 43:42-46, 2005

26. Pereira CU, de Jesus RM: Epidemiologia do traumatismo raquimedular. J Bras Neurocirug 22:26-31, 2011

27. Pham M, Bäumer P, Meinck H-M, Schiefer J, Weiler M, Bendszus M, et al: Anterior interosseous nerve syndrome: fascicular motor lesions of median nerve trunk. Neurology 82:598-606, 2014

28. Schreiber JJ, Byun DJ, Khair MM, Rosenblatt L, Lee SK, Wolfe SW: Optimal axon counts for brachial plexus nerve transfers to restore elbow flexion. Plast Reconstr Surg 135:135e-141e, 2015

29. Sunderland S: The intraneural topography of the radial, median and ulnar nerves. Brain 68:243-299, 1945

30. Sungpet A, Suphachatwong C, Kawinwonggowit V: Onefascicle median nerve transfer to biceps muscle in C5 and C6 root avulsions of brachial plexus injury. Microsurgery 23:10-13, 2003

31. Tsai YA, Chuang TY, Yen YS, Huang MC, Lin PH, Cheng H: Electrophysiologic findings and muscle strength grading in brachioplexopathies. Microsurgery 22:11-15, 2002

32. van Zyl N, Hahn JB, Cooper CA, Weymouth MD, Flood SJ, Galea MP: Upper limb reinnervation in C6 tetraplegia using a triple nerve transfer: case report. J Hand Surg Am 39:1779-1783, 2014

33. Zancolli EA: Midcervical tetraplegia with strong wrist extension: a two-stage synergistic reconstruction of the hand. Hand Clin 18:481-495, vii, 2002

\section{Disclosures}

The authors report no conflict of interest concerning the materials or methods used in this study or the findings specified in this paper.

\section{Author Contributions}

Conception and design: Bertelli. Acquisition of data: both authors. Analysis and interpretation of data: Bertelli. Drafting the article: both authors. Critically revising the article: Bertelli. Reviewed submitted version of manuscript: both authors. Approved the final version of the manuscript on behalf of both authors: Bertelli.

\section{Supplemental Information \\ Videos}

Video 1. https://vimeo.com/170022272.

Video 2 . https://vimeo.com/170022743.

Video 3. https://vimeo.com/170022840.

Video 4. https://vimeo.com/170022908.

\section{Correspondence}

Jayme A. Bertelli, Rua Newton Ramos 70, Apto 901, Florianópolis-SC, 88015395, Brazil. email: drbertelli@gmail.com. 